\title{
ATMOSPHERIC ENVIRONMENT DETECTION METHOD BASED ON MULTI-ROTOR UAV PLATFORM
}

\author{
Ao Shen ${ }^{1,2}$, Shudao Zhou ${ }^{1,3, *}$, Shuling Peng ${ }^{1}$ \\ ${ }^{1}$ College of Meteorology and Oceanography, National University of Defense Technology, Nanjing 211100, China \\ ${ }^{2}$ Unit 94270 of PLA, Jinan 250117,China \\ ${ }^{3}$ Collaborative Innovation Center on Forecast and Evaluation of Meteorological Disasters, Nanjing University of Information \\ Science \& Technology, Nanjing 210044, China
}

Commission III, WG III/8

KEY WORDS: Multi-rotor UAV, Atmospheric detection, Flow field, Experiment

\begin{abstract}
:
For the method of atmospheric detection based on multi-rotor UAV, the effects of flight safety and airflow on detection accuracy are analyzed. The detector mounted on the multi-rotor drone should be highly symmetrical and the center of gravity should be on the same vertical line as the center of gravity of the drone. Mounting the detector above the drone's fuselage will reduce the stability of the drone, and lowering it will improve stability, but for models with high stability, placing it below will affect the maneuverability of the drone. The airflow interference in the area where the detector can be placed near the fuselage is related to the drone: the faster the rotor speed, and the closer the rotor is to the fuselage, the stronger the airflow interference. At the same time, the influence of airflow disturbance on temperature and humidity has a gradual process. Waiting for a period of time after starting the drone can change the error into a fixed error for post-processing. This method was used to detect a pollution process and achieved good results.
\end{abstract}

\section{INTRODUCTION}

The meteorological conditions of the atmospheric boundary layer play an important role in the formation, maintenance and dissipation of pollutants. The vertical synchronization observation of the meteorological elements and pollutant concentration distribution in the atmospheric boundary layer is sufficient to fully understand the characteristics of the pollution process. It is of great significance to effectively prevent pollution weather and provide timely and optimal emergency management measures and decision-making basis. The current detection experiments mainly use air-seeding, mooring, wind profiler, and meteorological towers. These methods are less flexible, greatly affected by the environment and difficult to obtain the three-dimensional structural characteristics of the pollution process, which cannot meet the current theoretical analysis and actual conditions.

The method based on the rotorcraft UAV platform to detect the elements in the atmospheric boundary layer has many advantages. On the one hand, the UAV has the advantages of flexible flight, low cost, convenient take-off and landing, and fixed-point hovering in the air. The method is more flexible, and the flight route can be continuously adjusted according to the characteristics of meteorological elements of the polluted weather; On the other hand, it can acquire more continuous data than the fixed point detection to increase the data density of the space.

At present, the method of detecting the atmospheric environment based on the multi-rotor UAV mainly adopts the installation of the detecting instrument on the multi-rotor UAV, and taking advantage of the multi-rotor UAV, the detection instrument is carried over the detection area for detection. However, the detection instrument is usually suitable for the ground environment. The following points must be considered when directly mounting on a multi-rotor UAV:
(1) Flight safety. Installing other equipment on a multi-rotor drone will change the weight and center of gravity of the drone and may have an impact on flight safety.

(2) Influence of airflow interference on detection accuracy. Because the rotor rotation of the drone produces a powerful airflow, it will affect the detector on the drone.

(3) The precautions of the detection instrument during the detection of the multi-rotor UAV.

This article will analyze the detection methods in the above aspects.

\section{FLIGHT SAFETY}

The multi-rotor UAV is generally symmetrical in structure, and the lift is generated by the rotation of the rotor. Changing the rotational speed of the corresponding rotor can change the magnitude of the lift acting on the drone to control the drone. Synchronously changing the speed of the front and back paddles can change the torque received by the drone and realize the yaw action of the drone. Therefore, the center of gravity of the installed sensor should be as close as possible to the center of the drone, and the structure should be as symmetrical as possible.

In addition, the height of the center of gravity of the multi-rotor drone and the angle of the rotor tilt will also have different effects on the performance of the drone. For example, the center of gravity of some multi-rotor drones is closer to the plane of the rotor, even higher than the plane of the rotor, as shown in Figure 1(a). And some drones have a center of gravity that is significantly lower than the rotor plane, as shown in Figure 1(b) Some drone rotors have a certain inclination inside or outside, as shown in Figure 1(c). In general, lowering the center of gravity will increase the stability of the drone (while reducing flexibility). In general, lowering the center of gravity and tilting the rotor inward increases the stability of the drone (while reducing flexibility). And, the increase in the number of rotors

\footnotetext{
* Shudao Zhou, zhousd70131@ sina.com
} 
will also increase the stability of the drone. For multi-rotor UAVs with low stability, the heavier detection instruments should not be installed above the fuselage body, otherwise the overall center of gravity will be further increased, posing a safety hazard. For highly stable drones, the instrument can choose the installation location as needed. But at the same time, if the detection instrument is installed under the drone with very high stability, the flexibility will be degraded. If the drone needs to change the posture frequently during the detection process, this method will lead to excessive consumption and affect the endurance time.

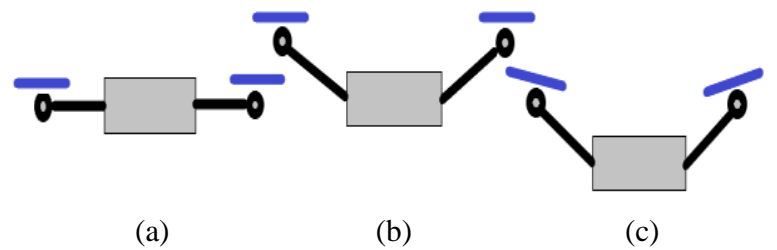

Figure 1. Multi-rotor drones of different structures

In general, the trend is shown in Table 1.

Table 1 . Trends of stability and flexibility,

\begin{tabular}{|c|c|c|c|}
\hline $\begin{array}{c}\text { Number of } \\
\text { rotors }\end{array}$ & $\begin{array}{c}\text { Small } \\
\text { quantity }\end{array}$ & $\begin{array}{c}\text { Increasing } \\
\text { quantity }\end{array}$ & $\begin{array}{c}\text { Large } \\
\text { quantity }\end{array}$ \\
\hline $\begin{array}{c}\text { Height of } \\
\text { gravity }\end{array}$ & $\begin{array}{c}\text { High center } \\
\text { of gravity }\end{array}$ & $\begin{array}{c}\text { Increasing } \\
\text { gravity }\end{array}$ & $\begin{array}{c}\text { Low center } \\
\text { of gravity }\end{array}$ \\
\hline Rotor angle & Tilt outward & Angle change & Tilt inward \\
\hline Stability & \multicolumn{3}{|c|}{ Increment } \\
\hline Flexibility & \multicolumn{3}{|c}{ Decrement } \\
\hline
\end{tabular}

\section{AIRFLOW INTERFERENCE}

Because the rotor rotation of the drone produces a powerful airflow, it will affect the detector on the drone. In order to analyze the influence of airflow on the detector, in this paper, the computational fluid dynamics method is used. This paper selects ANSYS simulation software. ANSYS software is a commonly used finite element analysis simulation software. It has a complete set of finite element preprocessing, calculation and analysis modules, and is widely used in fluid mechanics, thermodynamics, electromagnetics and other fields.

Firstly, the air in the continuous medium is discretized by using the finite element analysis method. Use the ICEM module in ANSYS software for meshing. According to the characteristics of the multi-rotor UAV, the fluid velocity near the rotor is fast, and the mesh needs to be encrypted, while the mesh of the rest can be relatively sparse. The selection model and meshing are shown in Figure 2.

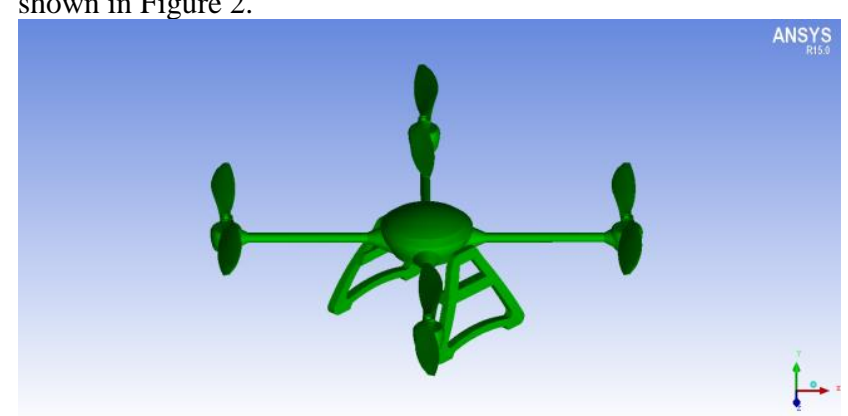

(a)

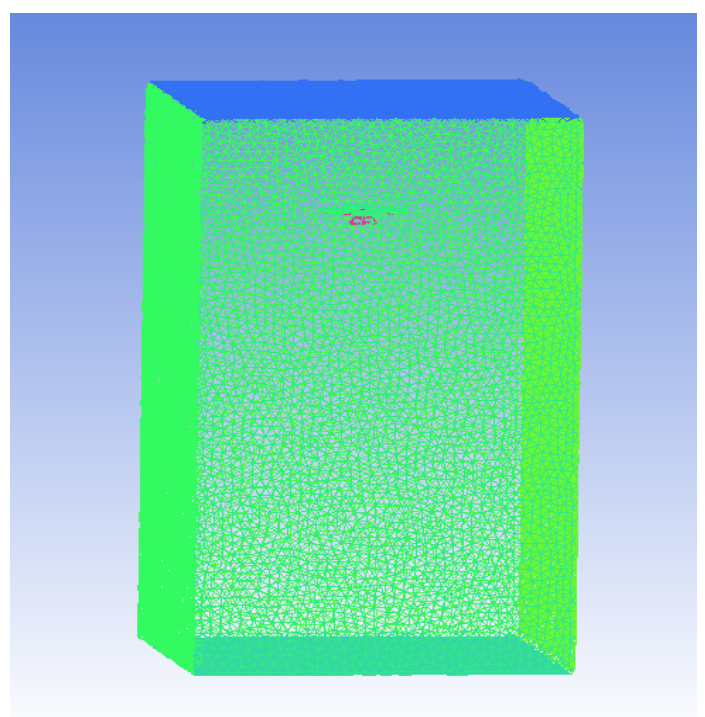

(b)

Figure 2. Model and meshing

By using the basic equations of mass conservation, energy conservation and momentum conservation, each discrete gas is described to form a large equation system. Then use the appropriate transfer equation to close the equations. The fluid conservation equation is expressed as follows:

$\frac{\partial \rho}{\partial t}+\frac{\partial}{\partial x_{\mathrm{i}}}\left(\rho u_{\mathrm{i}}\right)=0$

$\frac{\partial}{\partial t}\left(\rho u_{i}\right)+\frac{\partial}{\partial x_{j}}\left(\rho u_{i} u_{j}\right)=-\frac{\partial \rho}{\partial x_{i}}+\frac{\partial}{\partial x_{j}}\left(\mu \frac{\partial u_{i}}{\partial x_{i}}-\rho \overrightarrow{u_{i}^{0} u_{j}}\right)+S_{i}$

$\frac{\partial}{\partial t}(\rho \emptyset)+\frac{\partial}{\partial x_{j}}\left(\rho u_{j} \phi\right)=\frac{\partial}{\partial x_{j}}\left(\Gamma \frac{\partial \emptyset}{\partial x_{j}}-\rho \overline{u_{j}^{g} \emptyset}\right)+s$

Where $\rho$ is the density; $u$ is the flow velocity; $\mu$ is the fluid viscosity; $\mathbb{\Gamma}, \emptyset$ is constant, $S$ is the original item.

This paper selects the $\mathrm{S}-\mathrm{A}$ model, the transport equation is a function of the turbulent kinetic energy $k$ :

$$
\begin{aligned}
\frac{\partial(\rho k)}{\partial t}+\frac{\partial\left(\rho \hbar u_{i}\right)}{\partial x_{i}} & =\frac{\partial}{\partial x_{j}}\left[\left(\mu+\frac{\mu_{t}}{\sigma_{k}}\right) \frac{\partial k}{\partial x_{j}}\right]+\mu_{t}\left(\frac{\partial u_{i}}{\partial x_{j}}+\frac{\partial u_{j}}{\partial x_{i}}\right) \frac{\partial u_{i}}{\partial x_{j}} \\
& -\rho C_{D} \frac{k^{3 / 2}}{l}
\end{aligned}
$$

Where $\sigma_{k}, C_{D}$ are empirical constant. $\mu_{t}$ is turbulent viscosity. Then use the computer to carry out the computational simulation, and finally simulate the flow field characteristics of the multi-rotor UAV during flight. The distribution of airflow and flow rate is shown in Figure 3. It can be seen that the area with a large influence on the airflow is mainly located below the rotor, and the flow velocity below the fuselage is slightly larger than that above the fuselage.

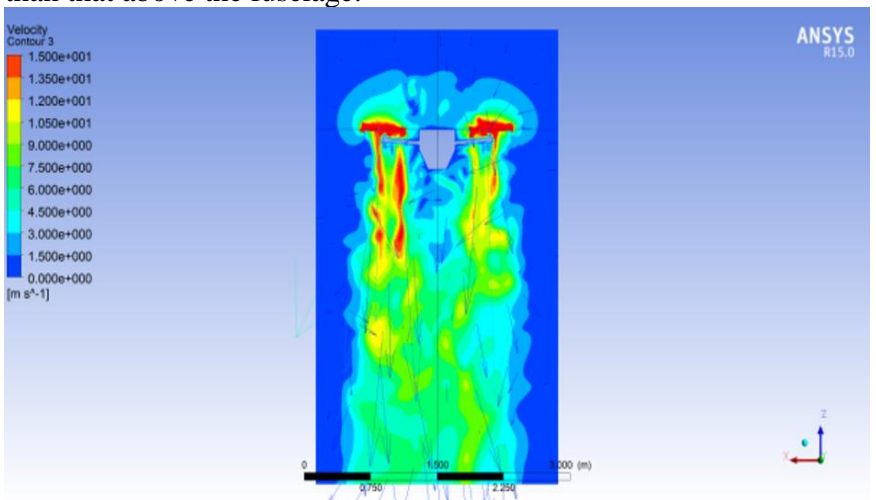

Figure 3. The distribution of airflow and flow rate 
Change the rotor speed, drone size, and rotor tilt angle by controlling the variable method, it can be found that: (1) the airflow near the fuselage is positively correlated with the rotor speed; (2) the airflow near the fuselage is negatively correlated with the distance from the rotor to the center of the fuselage; (3) The airflow interference area is related to the angle of the rotor and the horizontal plane. When the rotor is tilted inward, the airflow above the fuselage of the drone has a greater influence. When the rotor is tilted horizontally or outwardly, the airflow under the fuselage of the drone has a greater influence, as shown in Figure 4.

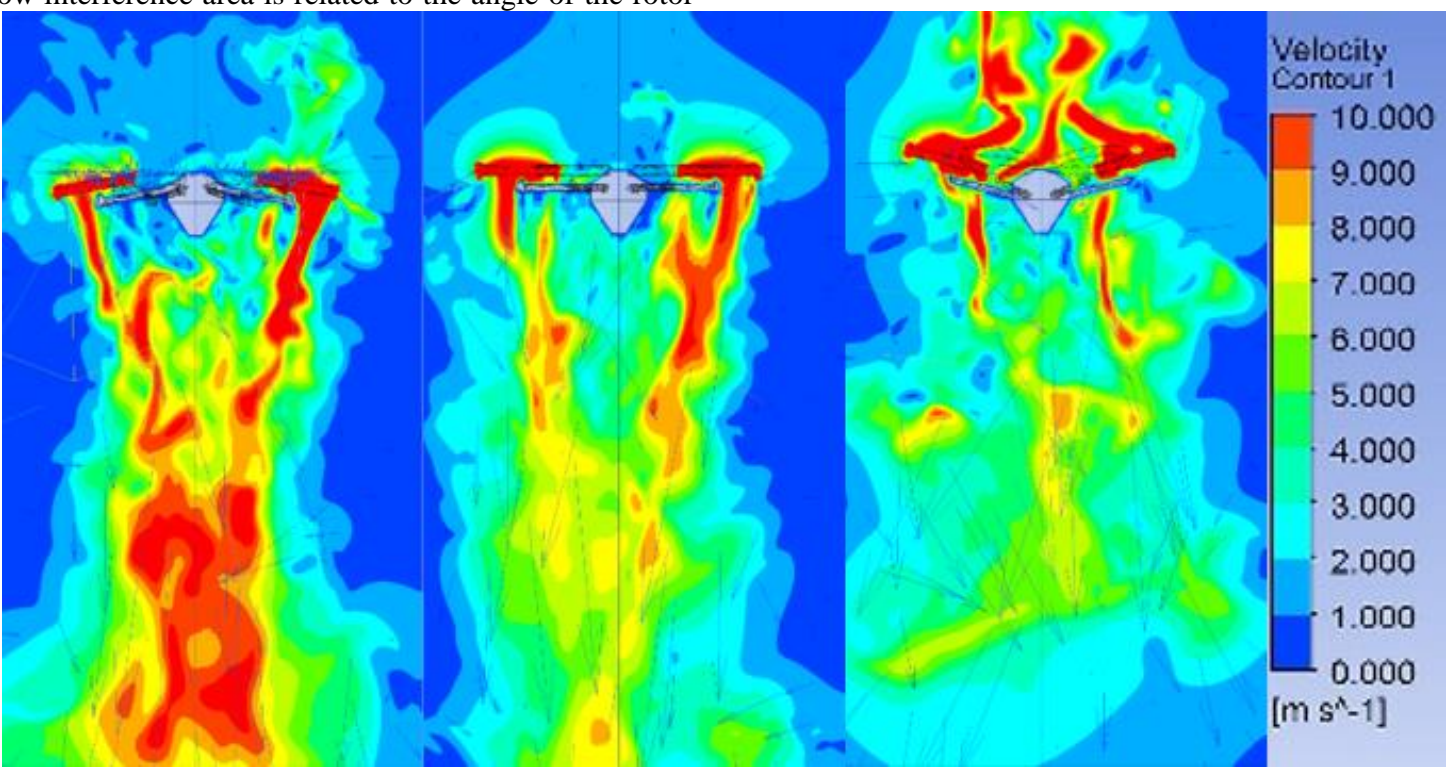

Figure 4 Flow field when the rotor is tilted outward/horizontal/inward

\section{PRECAUTIONS OF DETECTOR}

In the detection experiment, the speed required for the drone to take off and the shape of the drone cannot be changed, and the detecting instrument can only be placed in the less influential area for the drone used. Figure 5 shows the results of the measured data in different placement areas. It can be seen from the figure that when the rotor of the UAV rotates, the temperature and humidity measured data change significantly, and the right placement position can reduce the error of data change. At the same time, it can be seen that when the rotor rotates, the temperature and humidity change gradually, but tend to be stable after a certain time. Therefore, in the detection, the UAV can be hovered for a period of time, and the formal detection experiment is started after the data of detector is stabilized, which is convenient for correcting the error.

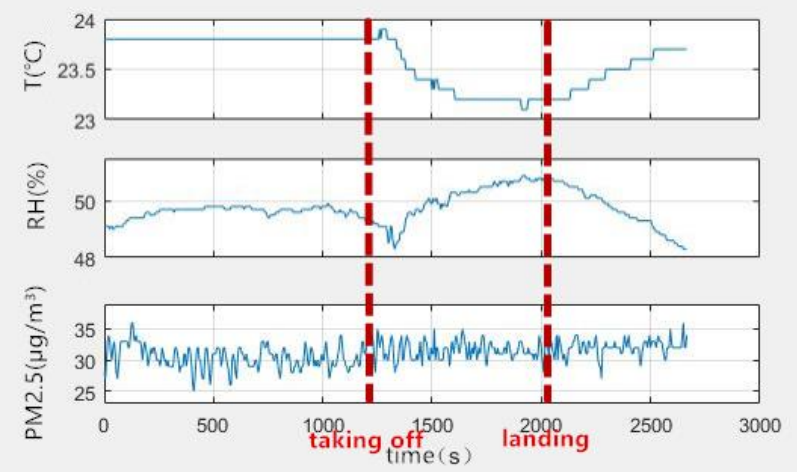

(a)

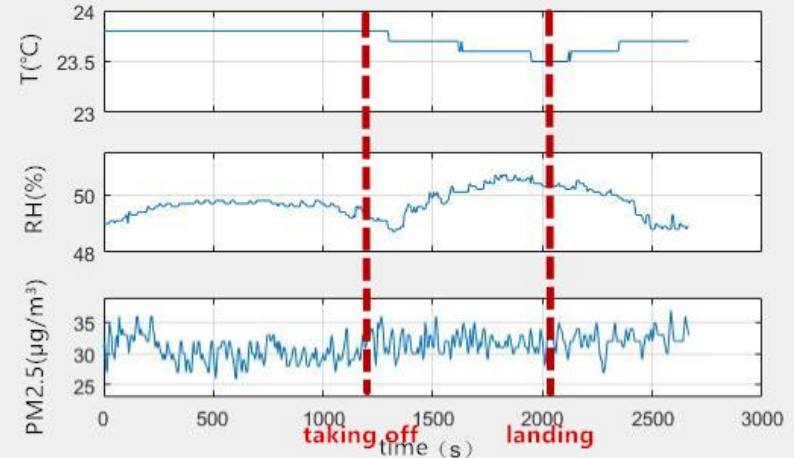

(b)

Figure 5 Detector data measured at different placement positions

\section{ATMOSPHERIC DETECTION USING A MULTI- ROTOR DRONE}

Based on the above method, a detection test was conducted on December 3-4, 2017 at the Xianlin Campus of Nanjing University $\left(118^{\circ} 57^{\prime} \mathrm{E}, 32^{\circ} 06^{\prime} \mathrm{N}\right)$. In the experiment, the UAV detected the temperature, relative humidity and PM2.5 concentration of $1000 \mathrm{~m}, 900 \mathrm{~m}, 800 \mathrm{~m}, \ldots 100 \mathrm{~m}, 0 \mathrm{~m}$ height layer. A total of 12 voyages were carried out during the test, and aerial surveys were conducted every 3 hours. In order to facilitate the distinction, the voyages are divided into three groups. Group A is the daytime voyage on the $3 \mathrm{rd}$, Group B is the voyage from the 3rd night to the 4th morning, and the $\mathrm{C}$ group is the 4th day voyage. The voyage information is shown in Table 2. Figure 6 shows the vertical profile of PM2.5 concentration, temperature and humidity measured for these 12 voyages. It can be seen that the method of detecting the atmospheric environment based on the multi-rotor UAV can conveniently obtain the vertical distribution characteristics of meteorological elements and pollutant concentrations. Combining this method with others 
allows for a more comprehensive study of the formation and transport of contaminants.

Table 2 Voyage information and ground PM2.5 concentration and weather conditions

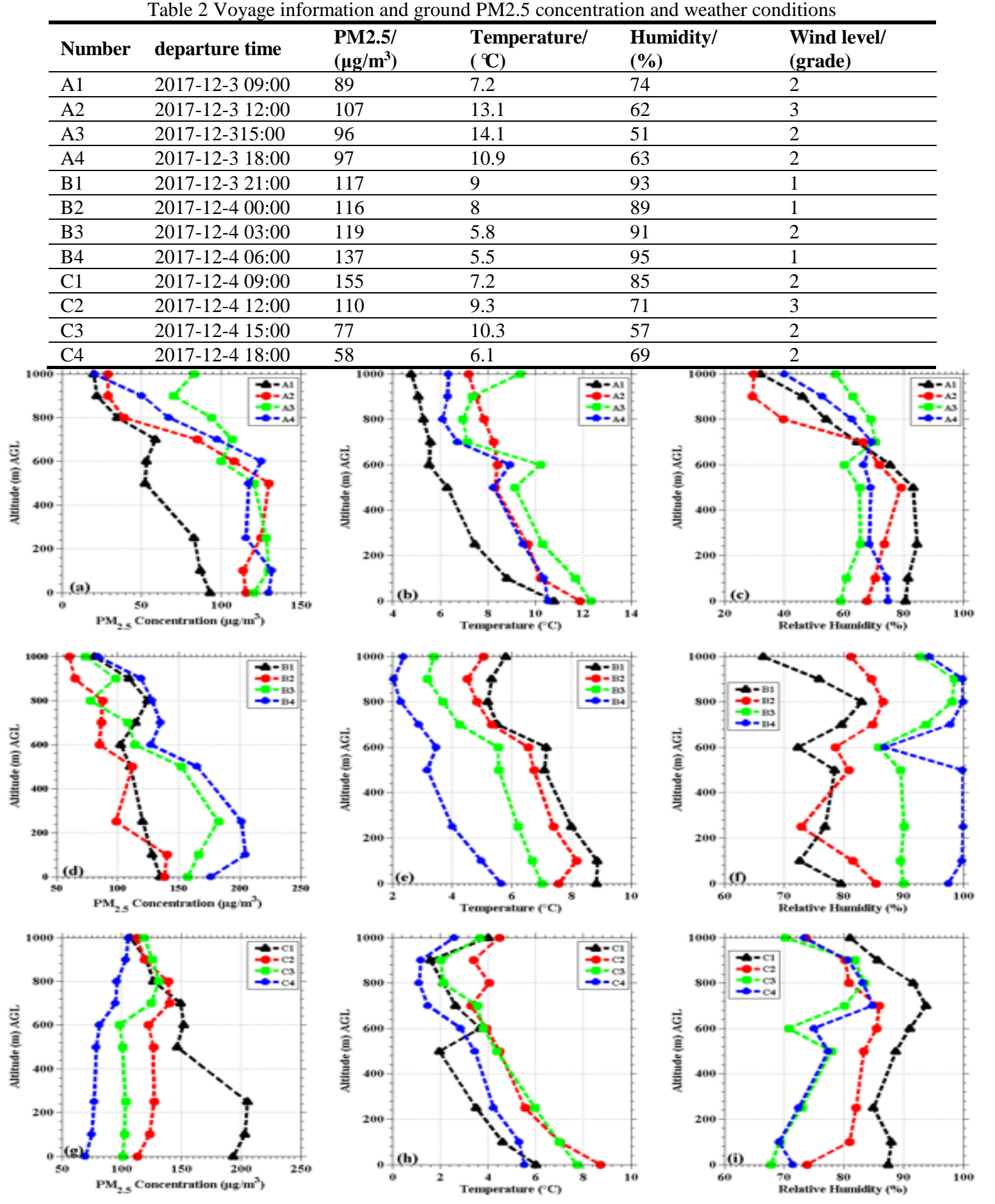

Figure 6 Vertical profile of PM2.5 concentration, temperature and relative humidity

\section{CONCLUSION}

For the method of detecting the atmospheric environment based on a multi-rotor UAV, the location where the detector is placed requires a comprehensive analysis of flight safety and airflow disturbances. At the same time, the UAV can be hovered for a period of time before the official detection is started, which improves the accuracy of the detection.

\section{ACKNOWLEDGEMENTS}

This research was supported by the National Natural Science Foundation of China (91544230, 41775039, 41775165)

\section{REFERENCES}

Shen A, Zhou S, Wang M, et al. Simulation and Optimization Design of Atmospheric Detection Equipment Layout Based on UAV[J]. Computer Measurement \& Control, 2018.(in Chinese) 
Shudao Zhou, Shuling Peng, Min Wang, Ao Shen, Zhanhua Liu. The Characteristics and Contributing Factors of Air Pollution in Nanjing: A Case Study Based on an Unmanned Aerial Vehicle Experiment and Multiple Datasets[J]. Atmosphere 2018, 9, 343.

Zheng Y, Yang S, Liu X, et al. The computational fluid dynamic modeling of downwash flow field for a six-rotor UAV[J]. Frontiers of Agricultural Science and Engineering, 2018, 5(2).

Guan Z Y, Jie L I, Yang H, et al. Research on Attitude Estimation of Micro UAV Based on Sparse Line Optical Flow Field[J]. Acta Armamentarii, 2014, 35(11):1851-1859.

Ryan A, Zennaro M, Howell A, et al. An overview of emerging results in cooperative UAV control[C]// Decision and Control, 2004. Cdc. IEEE Conference on. IEEE, 2004:602-607 Vol.1.

Ross S, Melik-Barkhudarov N, Shankar K S, et al. Learning monocular reactive UAV control in cluttered natural environments[C]// IEEE International Conference on Robotics and Automation. IEEE, 2013:1765-1772.

Chen J, Zhang Y. Using HF-Doppler Detector to Analyze Infrasonic Disturbances[C]// International Conference on Microwave and Millimeter Wave Technology. IEEE, 2007:1-2.

Abedin M N, Refaat T F, Singh U N, et al. Multicolor focal plane array detector technology: a review[J]. Proceedings of SPIE - The International Society for Optical Engineering, 2003. 